\title{
ARTIGOS
}

\section{LES TRANSFIGURATIONS DE LA NOTION DE PHYSIS ENTRE HOMÈRE ET ARISTOTE}

\author{
Lambros Couloubaritsis* \\ lambros.Couloubaritsis@ulb.ac.be
}

RÉSUMÉ L'auteur analyse la pluralité et la consistance de la notion de physis, mais aussi les limites de sa valorisation comme une notion éminente et absolue, à partir de quelques penseurs entre Homère et Aristote. Il approche le problème de la nature dans l'ordre du devenir et de l'évaluation qu'en font les divers penseurs, avant sa réhabilitation par la Physique d'Aristote, point culminant de la science antique, qui a déterminé la pensée européenne pendant des siècles. Sensible à la pluralité des transfigurations du concept dans des contextes divers, l'article vise à éclairer la polysémie de physis.

Mots clés Physis, nature, Homère, Pré-socratiques, Platon, Aristote

RESUMO O autor trabalha a pluralidade e a consistência da noção de physis, mas também os limites de sua valorização como uma noção eminente e absoluta, a partir de alguns pensadores entre Homero e Aristóteles. Ele aborda o problema da natureza no âmbito do devir e das diferentes avaliações que fazem os pensadores, antes de sua reabilitação na Física de Aristóteles, ponto culminante da ciência antiga, que determinou o pensamento europeu durante séculos. Sensivel à pluralidade de transfigurações do conceito em contextos diversos, o artigo visa esclarecer a polissemia de physis.

Palavras-chave Physis, natureza, Homero, Pré-socráticos, Platão, Aristóteles

* Professeur à l'Université Livre de Bruxelles et éditeur de la Revue de philosophie ancienne. Recebido em 12/05/2009 e aprovado em 11/07/2009.

KRITERION, Belo Horizonte, nº 122, Dez./2010, p. 349-375. 


\section{Lectures controversées de physis/natura}

L'histoire antique de la notion de physis a atteint son point culminant avec l'instauration d'une "science physique » par Aristote dans son texte Physikè akroasis ${ }^{1}$, qui a déterminé la pensée européenne dans sa formation, mais surtout à partir du $13^{\mathrm{e}}$ siècle, lorsque le christianisme médiéval a proposé sa propre version de l'aristotélisme, souvent contre celle des penseurs persans et arabes $^{2}$. Cette science, fondée sur une distinction entre monde sublunaire (où se déploie la physis) et monde supralunaire (qui la transcende, grâce au mouvement circulaire des étances célestes numériquement unes) a été ébranlée par Kepler à la suite de sa découverte des mouvements elliptiques, et par Galilée, Baliani et Newton grâce au principe d'inertie, articulé à travers un espace homogène et mathématisable ${ }^{3}$.

Or, comme la physique d'Aristote se situe dans l'espace qui sépare les pratiques de la physis avant l'institution d'une science (epistèmè) et la formation de la «physique» moderne, dite classique, on est tenté d'interpréter la notion de physis rétrospectivement dans l'éclairage de la science actuelle, au risque d'en faire une donnée préscientifique ou préhistorique des sciences de la nature. Cette interprétation prend comme critère les progrès inéluctables des sciences, en considérant que la seule valeur du passé est celle de son apport historique, propre au génie des pionniers qui mirent en œuvre les premiers balbutiements et, de ce fait, ne devrait pas influencer nos jugements sur sa pertinence et son statut scientifique.

Cette position a poussé certains interprètes, plus indulgents à l'égard d'Aristote, à considérer que sa physique est empirique, mais recèle les données importantes pour la métaphysique (P. Duhem, A. Mansion). D'autres, comme Heidegger, adoptant la réhabilitation depuis Nietzsche, des penseurs présocratiques, et plus spécialement ceux qu'Aristote appelle " physiologues », accordent une valeur éminente à la pensée de la physis. D'une façon différente, et selon des points de vue variables, W. Wieland (herméneutique), E. Berti

1 Cf. mon livre La Physique d'Aristote, Ousia, Bruxelles, $1997^{2}$ (1980), et mon Introduction et les notes dans Aristote, La Physique, trad. A. Stevens, Vrin, Paris, $2008^{2}$ (1999) .

2 Voir mon Histoire de la philosophie ancienne et médiévale, Grasset, Paris, 1998, pp. 937 ss.

3 Voir mes études, «La formulation du principe d'inertie par Baliani et la conception aristotélicienne du mouvement », Proceedings of the World Congress on Aristotle (Thessaloniki, 7-14 août 1978), T. II, Athènes, 1981, pp. 379-384, complété par «Purification par Baliani de la théorie ancienne du mouvement et généralisation du principe d'inertie », dans Coneption de la science : hier, aujourd'hui, demain. Hommage à Marjorie Grene, éd. J. Gayon et R.M. Burian, Ousia, Bruxelles, 2007, pp. 261-272 ; ainsi que « L'avènement de la science dans l'antiquité et la modernité », Sartoniana (Université de Gand), 11, 1998, pp. 43-65 et « De la pratique de l'Un d'Aristote à la formation de la science moderne ", dans De la science à la philosophie : Y a-t-il une unité de la connaissance?, éd. M. Cazenave, Albin Michel, Paris, 2004, pp. 379-421. 
(dialectique et problématicité) et moi-même (institution philosophique des principes) avons insisté sur le caractère principiel de la démarche d'Aristote.

La lecture de la pensée grecque instaurée par Heidegger a adopté la position la plus radicale, diamétralement opposée à celle des scientistes, en considérant la physis comme relevant d'une démarche originaire, plus profonde, occultée ou déviée par la scientificité introduite par Platon pour les intelligibles et élaborée par Aristote pour le devenir et le sensible. La traduction latine de physis par natura (comprise par Heidegger comme signifiant « naître » et « naissance ») a fait le reste, parce qu'elle aurait détruit «l'authentique force de l'appellation philosophique », en détournant le contenu originel du mot grec $^{4}$. Cette lecture de la pensée grecque s'accorde avec le proverbe chinois qui dit que la source désapprouve toujours l'itinéraire du fleuve, et cautionnez l'approche « pré-scientifique » au sens d'un rapport plus essentiel de l'homme au monde, préalable à toute approche scientifique des choses. Heidegger prolonge la phénoménologie husserlienne, qui situe en deçà de tout énoncé scientifique un rapport aux choses qui est " pré-scientifique », comme dans le cas du rapport intentionnel de proximité au «rouge», préalable à sa formulation sur le mode d'une longueur d'onde. Par un glissement de sens, il interprète la physis, d'une façon originaire, à partir de l'être de l'étant dans le sens d'une venue dans la présence qui aussitôt l'occulte la provenance, en laissant place à l'étant, formulable selon différents modes, y compris celui de la science physique. En tant que venue dans la présence, la physis se manifeste sur le mode d'une éclosion, d'un lever (comme on dit « le soleil se lève ») ou, selon une traduction plus poétique (G. Kahn), comme épanouissement, — qui enrichit le sens de Aufgehen, et qui me semble plus proche du terme grec. Mais il y a plus.

Intégré dans la thèse d'après laquelle le déploiement historique de la philosophie s'accomplit comme l'histoire de l'être, l'avènement de la physis représenterait, selon Heidegger, un moment antérieur à l'irruption des Idées (Platon) et de l'energeia (Aristote), donc aussi à la formation des données scientifiques édifiées par Newton. Tous ces moments constituent, avec d'autres, des figures successives de l'être sur le mode de l'étant, qui tracent l'itinéraire de la métaphysique jusqu'aujourd'hui. Ils ont abouti au triomphe de la technique contemporaine, comme concrétisation du platonisme dans les objets techniques. De sorte que la physis originaire s'accorderait à la fécondité méditative de la pensée, identifiée au logos comme conduisant au mode le plus 
propre des choses, proche du poétique ${ }^{5}$, et oblitérée par les figures successives de l'histoire de la philosophie (c'est-à-dire de la métaphysique) avec, comme point culminant à notre époque, l'irruption des étants techniques, soumis à la pensée calculatrice.

Ce type d'interprétation comprend la notion de physis en opposition avec son appropriation latine, et relativement aux notions de natus (naissance, croissance), de nascor (1. naître, se produire, venir au monde ; 2. tirer son origine commencer à être, provenir) et de natura compris au sens de « naissance ». Or, il semble que ce dernier terme est utilisé par les Romains en parallèle avec ingenium (qualité, propriété naturelle, disposition) ${ }^{6}$, mais sans interférence avec « naissance ${ }^{7}$. Carlos Lévy écrit que l'acception la plus répandue pour les premiers emplois de natura est " "caractères innés", "dispositions résultant de la naissance", sens également fréquents pour physis $»^{8}$. L'expansion de l'usage de natura à Rome est due au développement de la culture grecque, si bien que ce terme serait un artefact, « un calque sémantique » (A. Pellicer) reproduisant les caractéristiques du terme grec. Relativement à l'étymologie, physis et natura signifieraient tout au plus « un surgissement et le résultat de celui-ci, l'unique différence étant que l'un se réfère au monde de la végétation, tandis que l'autre s'applique au monde animal $»^{9}$.

Ces observations s'écartent pourtant des dictionnaires, qui mettent l'accent sur l'ambiguïté du terme, en retenant, d'une part, le sens « naissance », " génération », « action de mettre au monde », et d'autre part, celui de « essence », « constitution «, « caractère », « penchant inné », « qualité », " propriété »). Peu importent ici les appropriations romaines de physis. De toute façon, d'une manière ou d'une autre, elles trouvent leur ancrage dans la pensée pré-aristotélicienne, même s'il convient d'en nuancer le sens.

En effet, très tôt, phyein signifie « pousser » au sens d'une plante qui pousse (phyton), ce qui permettrait aux notions de physis/natura de rendre l'idée de «forces vitales et créatrices » dans un être ou dans le Tout ou une

5 «Les Grecs, écrit Heidegger, n'ont pas commencé par apprendre des phénomènes naturels ce qu'est la physis, mais, inversement : c'est sur la base d'une expérience fondamentale poétique et pensante de l'être que s'est ouvert à eux (aufgrund einer dichtend-denkenden Grunderfahrung des Seins erschloss sich ihnen das), ce qu'ils ont dû nommer physis » (op. cit., p. 11 , trad. p. 27).

6 Ces informations proviennent de l'introduction de Carlos Lévy, à l'ouvrage collectif sur Le concept de nature à Rome. La physique, éd. C. Lévy, Presses de l'Ecole Normale Supérieure, Etudes de littérature ancienne, T. 6, Paris, 1996 , et qui se basent sur une thèse de A. Pellicer, Natura. Etude sémantique et historique du mot latin, Paris, 1966.

7 Il est utile de signaler ici, que l'ouvrage en question sur Le concept de nature à Rome, se place explicitement contre Heidegger, qualifié d'« adversaire " (op. cit., p. 7).

8 C. Lévy, «Philosopher à Rome », op. cit., p. 18-19.

9 Id. ibid., p. 19. 
partie du Tout, tout en signifiant, d'une façon plus statique, « essence » (ou « nature ») d'un être ou d'une chose, voire « constitution » ou, plus faiblement, « taille » et « propriété ». Cette polysémie, qui pointe une opposition entre un sens dynamique impliquant un devenir et son déploiement (point de vue diachronique), et un sens statique mettant en relief une consistance stable et solide (point de vue synchronique), ne doit pas nous troubler, mais au contraire nous interpeller et nous solliciter à dégager soit leur opposition inconciliable, soit leur connivence secrète, soit encore leur complémentarité explicite.

Le premier sens concerne le « devenir » ou, mieux, le « déploiement» et l' "épanouissement » (dans la mesure où ce qui devient apparait comme un multiple organisé et non seulement successif), tandis que le second concerne une « propriété » ou, mieux, une « constitution» qui traduit une « essence ». Le plus souvent ces perspectives s'opposent à ce qui les excède ou au contraire à ce qui leur est inférieur. Le premier cas peut être illustré par le monde supralunaire supérieur où règne un mouvement circulaire perpétuel en dehors de tout devenir, ce qui amoindrit la valeur de la physis face aux choses divines (astres) ; et le second cas concerne la loi conventionnelle (nomos), l'art/technique (technè), le hasard (tychè),l'habitude (hexis) qui, à l'inverse, confèrent à la physis une consistance plus grande, tantôt à cause de sa puissance intrinsèque et tantôt grâce à la vie (cas des vivants). Ces nuances montrent que la physis n'est pas toujours globalisante, rapportée au Tout, et ne peut contribuer à défendre l'idée d'après laquelle l'histoire antique de la physis a un dénominateur commun qui rapproche les philosophes anciens, du moins avant le néoplatonisme ${ }^{10}$. Comme je l'ai montré ailleurs, Heidegger a perdu de vue que la physique du mélange, introduite par Parménide, et prolongée par Empédocle, les atomistes et Anaxagore s'est édifiée contre la notion de physis au sens d'épanouissement, parfois même, comme nous le verrons, contre cette notion même (chez Anaxagore) ${ }^{11}$. Une étude attentive de la pensée antique révèle une profusion de pensées, irréductibles entre elles, mais en même temps la présence d'une consistance plus grande de la notion de physis, que celle qui est relevée par la philologie, comme le prétend Carlos Lévy. C'est la pluralité des courants philosophiques qui fait la richesse de l'Antiquité, et ce sont les transfigurations des concepts dans leur contexte propre qui éclaire la polysémie de physis.

10 La thèse globalisante a été défendue par Heidegger, qui dit que « l'étant comme tel en Totalité les Grecs le nomment physis " (das Seiende als solches im Ganzen nennen die Grieschen phusis ), op. cit. p. 12, trad., p. 28.

11 «Les multiples chemins de Parménide », dans Etudes sur Parménide, éd. P. Aubenque, Vrin, Paris, T. II, 1987, pp. 25-43. 
Dans cette communication, je souhaite relever ce pluralisme et la consistance de la notion de physis, mais aussi les limites de sa valorisation comme une notion éminente et absolue. Il va de soi que je ne pourrais faire l'inventaire des usages de physis chez tous les penseurs entre Homère et Aristote. Je passerai sous silence trois grands courants : le pythagorisme (alors que Philolaos utilise souvent le terme physis), l'atomisme (qui comprend physis au sens de constitution) et la sophistique (où l'opposition entre physis et nomos prend divers sens, qui ont fait couler beaucoup d'encre). Je me limite à une dimension plus précise qui concerne l'histoire de la physis dans l'ordre du devenir et de l'évaluation qu'en font les divers penseurs, avant sa réhabilitation par Aristote. Cette histoire est, jusqu'à un certain point, extérieure ou parallèle à la philosophie de l'action et au traitement des mathématiques, qui constituent, depuis les Pythagoriciens et Platon la première science antique, dans laquelle il convient d'inclure l'harmonique, référence pour penser l'harmonie.

\section{Naissance, poussée ou épanouissement?}

Chez Homère, où les plantes ( phyta) sont souvent mises en valeur, phyein prend le sens de « pousser ». S'agit-il simplement d'un acte conforme à l'usage courant, lorsqu' on dit que telle plante pousse ? Ou, ce processus dit plus, c'està-dire un déploiement dans l'espace et en même temps un épanouissement qui manifeste la plante dans sa splendeur? Je crois que c'est ce plus qu'il convient d'envisager, car l'usage ponctuel du mot rend le sens restreint de " pousser » en l'enrichissant par d'autres éléments qui impliquent un «épanouissement».

Dans Iliade IV, lorsque Ajax frappe avec sa lance Simoïsios, celui-ci gît dans la poussière, et ressemble à un peuplier couché au sol herbeux d'un vaste marécage, où l'on voit la survivance de rameaux qui avaient poussé / s'étaient épanouis (pephyasi) sur la partie extrême ; il se dessèche aux bords du fleuve pour être taillé et utilisé comme la jante d'un char (v. 482-486). L'opposition ici entre l'épanouissement de l'arbre dans sa vigueur et son dessèchement après avoir perdu la vie, confirme qu'il faut utiliser, pour phyô, un sens fort. Ce texte voir que phyô dit plus qu'un surgissement et le résultat de celui-ci, comme le prétend Carlos Lévy. Ce plus est confirmé par l'Odyssée VI, dans l'épisode où la Nymphe apporte à Ulysse une hache de bronze et une fine doloire, tout en lui montrant le chemin vers la pointe de l'île, où « des arbres très haut avaient jadis poussé / s'étaient jadis épanouis (pephykei)... », mais qui étaient tous morts depuis longtemps, devenus secs et légers, pouvant flotter, ce qui permettait à Ulysse de construire un radeau (V, 232 ss.). Bref, l'arbre qui s'épanouit suppose, dans sa constitution, une consistance exprimée par son poids. Plus 
loin, il est question de deux oliviers, l'un franc et l'autre greffé qui, épanouis (pephyôtas) du même tronc, ne laissent pénétrés ni le vent, ni les brumes (V, 477 ss.). Dans ce nouvel exemple s'ajoute la vigueur des branches et de leurs feuilles. Victor Bérard (dans la collection Budé) traduit par «nés sur le même tronc », ce qui est trop faible, car il ne tient pas compte de la vigueur et de l'abondance qui explique le barrage que ces deux oliviers réalisent. Au livre VI, 114 ss., il traduit pephykasi tèlethoônta par « verger aux hautes ramures», et ta men phyein, par " fait bourgeonner les uns », alors qu'il est clairement question d'un épanouissement vigoureux et abondant. Je pourrais multiplier les exemples, mais je crois qu'il convient d'aller plus loin pour contextualiser davantage le problème selon le devenir, en l'occurrence le devenir comme déplacements (mythes des voyages) et le devenir dans l'ordre généalogique, qui est le propre, avec les mythes des voyages, des discours catalogiques à cette époque. Deux exemples, qui nous situent dans le domaine de l'étrangeté, peuvent nous aider à voir plus clair dans l'histoire de la notion de physis.

Dans l'Iliade XIV, lorsque les Achéens sont en déroute, Héra met tout en œuvre pour concilier les dieux. Elle quitte l'Olympe et se pose en Piérie et dans l'Emathie, avant de s'élancer vers les chaînes neigeuses de Thrace, pour arriver enfin, après diverses étapes, à Lemnos où elle trouve Sommeil (Hypnos), à qui elle demande d'endormir Zeus, ce qu'il refuse. Elle le relance en lui promettant comme épouse une Grâce, ce qui modère ses réticences. Il décide d'accompagner Héra et, en traversant Ida, ils atteignent ensemble le sommet d'un bois, où Zeus les aperçut. Alors Sommeil « monta sur un arbre géant, le plus haut qui a poussé/s'est épanoui (pephyyia) sur Ida et qui, à travers l'air, s'élève jusqu'à l'éther. Il se poste là, derrière un rideau de branches de pin, semblable (enaligkios) à l'oiseau qui chante d'une voix mélodieuse, que les dieux, sur les monts, appellent "chalcis", et les hommes, "cyminde" " (v. 226-291).

Il est clair que, d'après ce texte, le verbe phyô doit être compris dans le sens fort d'épanouir avec vigueur, plutôt que dans le sens faible de " naître » et même de « pousser ». Or, cet épisode est mis en scène au bout d'un récit où se succèdent différents moments d'un long voyage, mettant ainsi en œuvre un discours catalogique, associé à des narrations, des lieux, des temps, réunissant ainsi quatre axes d'analyse (discours catalogique, narrations mythologiques, topologie et chronologie), par lesquels j'ai qualifié le mythe, dans mes études $^{12}$. Nous découvrons, en quelques lignes, l'ampleur et la complexité de 
la pratique archaïque du mythe (en l'occurrence des mythes du voyage), mais aussi la consistance inouïe du verbe phyô, qui apparaît comme une donnée d'une structure narrative, plus étendue. Cette perspective qui contextualise l'usage de phyô au sens d'épanouissement est confirmée par un autre passage qui, lui, met en relief la parenté, donc aussi le discours généalogique, qui constitue un autre volet de la pratique du mythe que mes études ont promu.

Lorsque Homère décrit, dans Iliade VI, 118 ss., le combat entre Diomède et Glaucos, le premier interpelle le second en lui disant que s'il est un immortels, il ne souhaite pas le combattre, mais, par contre, s'il est un mortel se nourrissant du fruit de la terre labourée, alors il peut s'approcher afin qu'il arrive plus vite au terme fixé pour son destin. Glaucos lui répond : « Pourquoi me demander mon ascendance (geneè) ? Comme l'est l'ascendance (geneè) des feuilles, ainsi l'est celle des hommes. Les feuilles, les unes c'est le vent qui les épand sur le sol, les autres c'est la forêt vigoureuse qui les épanouit (phyei) quand advient (epigignetai) la saison printanière. De même pour l'ascendance (geneè) des êtres humains, l'une s'épanouit, l'autre cesse à un moment donné. Néanmoins, ajoute-t-il, si tu veux connaître davantage sur mon ascendance (geneèn) (...), sache qu'il existe une cité, Ephyre, au fond de l'Argolide, nourricière de cavales, où vivait Sisyphe... ». Ensuite, Glaucos mythifie sa généalogie, où son ancêtre Bellérophon, petit fils de Sisyphe, aimée d'Antée, l'épouse de Prœtos, roi d'Argos, subit une destinée funeste parce que, refusant ses avances, Antée l'accusa de l'avoir harcelée. Pour se venger, Prœtos l'envoi en Lycie, auprès de son beau-père, où Belléphron est bien accueilli jusqu'au jour où furent connus les signes funestes que Prœtos avait inscrits sur une tablette. Il subit une série d'épreuves mortelles, d'où il sortit néanmoins indemne, en tuant successivement la Chimère, les Solymes, les Amazones, les guerriers de Lycie, ce qui lui permis d'être réhabilité et se marier avec la fille du roi, qui lui donna trois enfant, dont l'un, Hippoloque fut le père de Glaucos. C'est lui, dit-il, qui « m'engendra et c'est de lui que je dis être né (etikte kai ek tou phèmi genesthai)» (v. 206). A quoi il ajoute : « en m'envoyant à Troie, il me recommandait d'être toujours le meilleur et surpasser tous les autres, de ne pas déshonorer la race de mes aïeux (genos paterôn), qui furent les plus braves, aussi bien à Ephyre que dans la vaste Lycie. C'est de cette ascendance et de ce sang que je me flatte d'être issu (tautès toi geneès te kai haimatos euchomai einai )» (v. 207-211).

l'étude « En cheminant entre mythe et philosophie: généalogie de la pensée de Lambros Couloubaritsis ", dans "Mais raconte-moi en détail... ", Mélanges de philosophie et de philologie offerts à Lambros Couloubaritsis, Vrin - Ousia,Paris - Bruxelles, 2008, pp. 37-54. 
Le texte est remarquable, car il associe discours généalogique et narration mythologique, qui inclut à travers les épreuves un autre discours catalogique (initiatique), où sont associés les axes topologiques et chronologiques, structurant la complexité du discours mythique. Or, si le discours généalogique circonscrit l'ascendance du héros, il est néanmoins éclairé par une analogie avec l'épanouissement des arbres de la forêt, où la provenance oppose épanouissement et extinction, et leur associe la valeur de la race, ce qui suppose que phyô est ici ambivalent, réunissant le devenir et la bonne constitution.

Cette constatation montre que dans la plupart des cas, la dualité existe, même si tantôt c'est l'une et tantôt l'autre qui domine. Ainsi, par exemple, quand Hésiode utilise le terme phyèn dans le mythe des races, à propos de la race (genos) d'argent (Travaux et Jours, v. 129), il dit que cette race n'était semblable à celle d'or, ni par la taille/constitution (phyèn), ni par la pensée. Comme il parle de naissance d'une nouvelle race, on peut lui associer une connotation dynamique, en liant, ici aussi, épanouissement et constitution. En réalité, les choses sont plus complexes, car la généalogie se déploie selon un catalogue issu du démembrement du réel en situant les éléments dans une succession, ce qui requiert un redressement pour reconstituer le réel ${ }^{13}$. Les sages d'Ionie vont réussir à modifier ce type de discours en le simplifiant, c'est-à-dire en considérant le devenir catalogique comme conforme à ce qui s'est passé.

Pour terminer avec la pensée mythique, je dirais que la sémantique de la physis est soumise au discours catalogique, dont le mode généalogique révèle une analogie subtiele avec l'épanouissement des plantes. Tout se passe comme si devenir des plantes et devenir généalogique des hommes favorisaitent un enchevêtrement plus actif, à condition que la généalogie discursive soit remplacée par des genèses successives de phénomènes naturels, intégrant davantage la notion de physis, en la liant au déploiement du devenir conformément au déroulement du temps. C'est ce glissement, correspondant à une transmutation du mythe, qui amorce la première transfiguration de la physis.

C'est, on le sait, Anaximandre et Héraclite qui confèrent à la notion de physis un rôle plus étendu, voire globalisant - qui a fort influencé la lecture extrême de Heidegger, mais dans un sens opposé au devenir proprement dit, qui suppose une succession de phénomènes physiques. Comme on le sait,

13 Pour une illustration de cette pratique, voir, en plus des études citées dans la note précédente, mon étude « Genèse et structure dans le mythe hésiodique des races ", dans Le métier du mythe, éd. F. Blaise, P. Judet de la Combe et Ph. Rousseau, Septentrion, Lille, 1996, pp. 475-518, qu'on peut compléter par mon livre La pensée de Parménide, op. cit., pp. 66 ss. 
Simplicius qui nous a légué le seul fragment significatif d'Anaximandre, en le situant dans son contexte. Je rappelle le texte:

« De ceux qui disent que le principe est un, mû et illimité, Anaximandre de Milet, fils de Praxiade, devenu successeur et disciple de Thalès, dit que l'Apeiron est une origine principielle (archèn) et un élément des choses présentes, faisant, le premier usage du terme archè. Il dit à son sujet qu'il n'est ni l'eau, ni rien d'autre de ce qu'on dit être des éléments, mais qu'il est une nature illimitée (physin apeiron), à partir de laquelle s'engendrent (ginesthai) les cieux et les univers qui s'ordonnent en eux ». Puis il cite Anaximandre (fr. 1): « les entités d'où il y a engendrement (genesis) des choses présentes (= des choses qui sont dans le présent), sont aussi celles vers où elles périssent (tèn phtora... ginesthai) selon la nécessité, car elles se rendent entre elles justice et réparation de leur injustice selon l'ordre du temps (kata tèn tou chronou $\operatorname{taxin}) »^{14}$.

L'expression « la nature de l'illimité » peut aussi être comprise comme «l'épanouissement de l'Apeiron ». Aristote écrit que « c'est l'Apeiron qui est le divin (kai tout'einai to theion), car il est immortel et incorruptible, comme le déclare Anaximandre et la plupart des physiologues ${ }^{15}$. Cela signifie que l'ordre du monde (univers) se produit à partir de cet Apeiron, par un épanouissement qui comporte des étapes successives. Hippolyte précise que la nature/épanouissement (physin) de l'Apeiron est perpétuel(le) (aidion) et ne vieillit pas (agèrô), et intègre ce propos dans une analyse proche de celle que propose plus tard Simplicius, c'est-à-dire qui situe entre l'Apeiron originaire et les choses qui sont dans le présent (eonta) le ciel et les mondes qui adviennent en lui.

Ces démarches réitèrent une suite catalogique qui modifie néanmoins la pratique archaïque du mythe, telle par exemple qu'on la rencontre dans la Théogonie d'Hésiode ${ }^{16}$. La succession implique un lien plus étroit entre les composantes et, en plus, s'accomplit sur le mode d'un épanouissement (physis), renversant ainsi le rapport archaïque entre genesis et physis. De ces textes, en effet, il ressort que la physis de l'Apeiron comme origine principielle (une, en mouvement et illimitée) déploie, selon des genèses successives et un ordre temporel, le ciel et les mondes qui sont en lui jusqu'à la genèse des choses multiples et éphémères qui sont dans le présent (eonta). Ce qui distingue donc cette démarche catalogique de celle d'Hésiode, c'est 
l'apport de l'expression physis apeiros. En tant que fonds unique (Un), origine permanente et infinie de genèses, l'Apeiron recèle un caractère perpétuel et immortel, sans possibilité de corruption et de périssement, accumulant tous ce qu'on attribuait au Divin suprême. Le fait qu'Anaximandre, comme la plupart des penseurs de la physis (physiologues), fait de ce fonds/fondement de tout, le véritable Divin, confère à la Physis comme fonds et épanouissement une primauté par rapport à la genèse. Par conséquent, l'association entre le Divin et la physis de l'Apeiron m'autorise à affirmer que la notion de physis est ambivalente: elle signifie à la fois « nature » (au sens de constitution) et « épanouissement ». Aristote est un témoin de cette idée, tout en l'infléchissant, lorsqu'il écrit que pour les penseurs de la physis (il parle d'Antiphon), " la nature et l'étance des étants naturels (hè physis kai hè ousia tôn physei ontôn) étaient, pour chaque chose, ce qui lui appartient en premier lieu et de façon immanente, mais non ordonnée par soi et en soi (arrythmiston kath 'heauto) ${ }^{17}$. Pour rendre plus proche ce texte àde l'ambivalence de la physis, il faudrait comprendre: " la physis, c'est-à-dire l'ousia... ». En tout cas, pour éclairer cette ambivalence on peut dire que physis signifie ici: épanouissement à partir d'un fondement un. La conjonction des genèses successives et d'un temps (chronos) signifiant le " temps conforme à des moments successifs », rend possible une description au moyen d'un discours catalogique qui comprend le monde actuel constitué à partir de sa genèse réelle, et non comme un moyen discursif (mythique) pour comprendre un monde inengendré et ordonné en séparant ses constituants et en les plaçant dans l'ordre d'une succession, dans un catalogue qui nécessite un redressement.

Tout se passe ainsi comme si un déroulement diachronique et un fonds synchronique se retrouvaient dans la notion de physis, de telle sorte que l'ordre du temps coïncide avec l'ordre de la constitution de l'univers avec le ciel, les mondes en lui et la multiplicité des choses éphémères qui aboutissent à l'ordre actuel de l'univers (synchronie). Autrement dit, les genèses successives n'étant plus celles du discours catalogique qui exprime l'ordre du monde toujours présent, mais celle du devenir proprement dit de la physis qui s'épanouit, la physis exprime le fonds qui se déploie et qui aboutit au multiple ordonné (kosmos) qui excède la physis, en réalisant d'autres activités, comme celles de la production et de l'action, c'est-à-dire de la technè et du nomos.

Cet excès qui attesterait une différence dans la physis même pose des difficultés philosophiques quant à l'unité et le statut du Tout, qui concerne 
aussi la difficulté d'expliquer le rapport entre l'Un et le Multiple, c'est-àdire d'expliquer comment l'Un peut produire du Multiple différent de lui, alors qu'il n'est qu'un. Cette faille, nous le verrons, conduit, d'abord, au rejet parménidien du devenir tel qu'il est façonné par les sages ioniens et, ensuite, au renversement platonicien qui situe l'Ame et la Technè divine comme préalables et plus essentielles que la nature matérielle. Cette dualité impensée dans l'unité de la Physis aboutira à une disqualification de la physis au sens d'épanouissement. Mais, pour mieux comprendre l'ampleur du problème, il convient d'apprfondir davantage la physis selon les penseurs ioniens à partir d'Héraclite.

Comme on le sait, le fr. 123, dit que « la physis aime à se cacher ». Physis est ici le Feu (qui remplace 1'Apeiron d'Anaximandre) qui se dérobe du fait qu'elle produit, par son épanouissement même, un monde dominé par le devenir et le multiple, — plutôt qu'une physis constituante dans son ensemble et qui se déroberait à l'homme dans le rapport qu'il entretient avec les choses qui entrent dans la présence (point de vue heideggerien). Cela signifie qu'en tant que fonds à partir duquel il y a épanouissement selon l'ordre du temps (chronos) et selon un rythme proportionné (logos), les choses qui font irruption progressivement sont éphémères et possèdent chacune un temps-de-vie (aiôn). C'est en produisant ce monde des choses en devenir que la physis se dérobe en s'appauvrissant pour faire émerger l'univers qui, par sa présence, dissimule le fondement.

Ce qui justifie cette interprétation qui met l'accent sur le devenir physique et non sur l'entrée dans la présence des choses, c'est la présence explicite du devenir dans le fr. 1 qui dit : « De ce Logos qui est toujours dans le présent (eontos aiei), les hommes sont sans intelligence, aussi bien avant de l'avoir entendu qu'après l'avoir entendu pour la première fois, car bien que toutes choses deviennent conformément au Logos, ils paraissent sans expérience quoique ils mettent en œuvre des paroles et des actions pareilles à celles que j'expose, divisant chaque chose selon la nature et la disant comme elle est ». Certes, on pourrait interpréter Logos, comme le font certains (par exemple J. Bollack) dans le sens de « langage », ce qui s'accorde davantage avec l'idée d'entendre. Mais dans ce cas, logos signifierait discours catalogique, ce qui est en dissonance avec l'assertion d'après laquelle ce qui est sage, c'est la recherche de l'Un-Tout. Bien plus, cela s'opposerait à l'idée qu'il faut dire les choses telles qu'elles sont et divisée conformément à la nature (kata physin), car, en réalité, tout discours catalogique requiert un redressement analogue à celui qu'exige le discours généalogique, pour rétablir l'ordre du monde. En fait, Héraclite utilise la polysémie du logos, ce qui déborde le langage, au 
profit des modes du « rassembler », y compris la raison et l'épanouissement constitutif. On peut dès lors considérer que le Logos est la Physis qui se dérobe bien qu'il soit toujours dans le présent, et que l'entendre signifie « prendre connaissance ». Dans ce cas, le devenir doit être exprimé selon un discours qui le dit adéquatement, mais avec comme horizon l'unité du Tout, en fonction des différents niveaux du logos: parole et raison personnelle, communication communautaire et rythme proportionné de la Physis qui règle de devenir permanent.

Il apparaît ainsi que la première transfiguration de la notion de physis avec les sages d'Ionie recèle des nuances, parmi lesquelles l'identification de la physis avec le logos confère un sens et un rythme (rythmos) à l'arrythmiston.

\section{Critique du devenir et naissance d'une physique du mélange}

Au moment d'aborder Parménide, on peut dire que dans la mesure où la notion de physis a investit la pensée des Ioniens d'une façon extensive (par une sorte de monisme matérialiste de l'épanouissement des choses multiples et éphémères à partir d'un fondement un) et promu le devenir selon une succession et un ordre conformément au temps, de nombreuses difficultés ont apparues. La première concerne la pertinence d'un devenir provenant d'une entité une (la physis comme Eau, Apeiron, Air, Feu). Comme je l'ai montré ailleurs ${ }^{18}$, parler d'un devenir du Multiple à partir d'une entité une pose un problème logique, l'Un ne pouvant produire que de l'Un, qui ne peut être, par surcroît, qu'identique à lui-même. Dès lors que cette option est rejetée, restent deux autres : ou bien il y a devenir à partir du néant, ce que Parménide refuse, ou le devenir doit envisagé à partir d'au moins deux entités, qui aboutissent à une physique nouvelle du mélange et de la séparation, au moyen de deux entités, le feu et la terre, analysés par Parménide à partir de la lumière et de l'obscurité. On l'aura compris, c'est à partir du la question du devenir plutôt que de la physis comme telle, que Parménide analyse la pensée ionienne, en ignorant ou occultant le point de vue d'une physis globale ou même partielle au sens d'épanouissement. Cela ne l'empêche pas de garder cette notion en réserve pour l'utiliser lorsqu'il étudie la pensée sur le plan physique, au fr. 16.

En fait, après avoir fondé la pensée continue sur l'Eon continu (« Ce qui est dans le présent » de façon permanente et absolue), il livre un certain nombre 
d'indices de sa théorie physique de la connaissance et de la vie, qui montrent que l'être vivant suppose toujours un mélange entre les deux « formes » selon des proportions variées. La pensée est d'autant meilleure et plus pure (beltiô kai katharôteran) que le chaud domine et excède, tandis que le mort se contente d'une seule des deux formes, la nuit et ses puissances propres, qui conservent encore et toujours l'activité sensitive, mais d'une façon pour ainsi dire obscure. L'équilibre entre la lumière et l'obscurité, tributaires du feu et de la terre, explique le mode physique sur lequel se déploie la pensée. Théophraste qui relève ces points a conservé un passage (fr. 16), qui dit : «Comme chaque fois, en effet, se trouve la co-pénétration des membres aux multiples mouvements flexibles, ainsi se dispose la faculté de penser chez l'homme ; car le même est, chez les hommes, en tous et en chacun, (la) nature (physis) des membres qui réfléchit,; en effet, c'est le plein qui fait la pensée $\rangle^{19}$.

Les résultats de mes recherches permettent d'interpréter le texte en relevant certaines thèses décisives pour éclairer la notion de physi ${ }^{20}$. Le terme krasis met en jeu une co-pénétration (ou inter-pénétration) de deux entités qui forment une unité dans laquelle elles sauvegardent néanmoins leur autonomie. Le mélange suppose une unité continue, en dépit de la multiplicité sous-jacente ${ }^{21}$. Le parallélisme établi entre le mélange des « choses » et la disposition de la faculté de penser (noos) fait voir que physis signifie mélange, ou mieux l'unité propre et constitutive du mélange, que l'on peut qualifier de « nature » dans un sens de « constitution » qui anticipe la notion d' «essence», comme lorsqu'on parle de la nature des choses.

Par suite, le corps, par le mélange qu'il implique, éclaire le fonctionnement physique du " penser ", lié à la notion de " même », qui qualifie l'Eon et le penser (fr. 3 et 8, 29-38). La présence de l'expression « le même » dans le fr. 16 est donc significative. Parménide considère que chacun et tous les hommes sont disposés de la même façon, selon un fonds qui est le même. Le « même » approfondit l'hekastote, qui manifeste la répétition concernant chaque mélange d'une « chose » donnée, c'est-à-dire le fait qu'il y a processus semblables pour le même type de « choses », et rend proche l'unité de fond (Eon) qui assure à l'homme son humanité et la structure physique de l'homme. Bref, le fr. 16 établit un lien entre les données de l'étude de « Ce qui est dans le

19 Je retiens successivement la leçon hekastote, au lieu des leçons hekastos ou hekastô ; polykamptôn, au lieu de polyplanktôn; et parestèken au lieu de paristatai.

20 Voir mon livre La pensée de Parménide, op. cit., pp. 505 ss.

21 Comme cela ressort encore de la pensée stoïcienne. Voir mon étude « Les pratiques hénologiques dans Le stoïcisme ancien », dans G. Romeyer-Dherbey et J. Gourinat (éd.), Le stoïcisme, Vrin, Paris, 2005, pp. $187-211$. 
présent » (Eon) et les données de la physique du mélange, grâce à cette notion de même, utilisée également pour la pensée et pour chacune des formes, la lumière et l'obscurité, mais impliquée également dans l'unité de deux. C'est ce dernier sens qu'on retrouve dans le fr. 16, où le même concerne également la loi propre à la physis. Cela permet aussi d'affirmer que c'est parce que «le même » constitue la loi de « Ce qui est dans le présent » et en même temps concerne la physis de l'homme, en tant qu'unité constitutive du mélange, qu'un rapport entre les deux parties du Poème devient possible. Le point de jonction se réalise ainsi par la médiation du penser, dont aussi bien l'Eon que la physis en sont la condition, mais autrement chacun d'entre eux. Après la fondation du penser et de la pensée dans la première partie du Poème, c'est au tour de la physique du mélange de fonder le penser.

Quant au sens de la notion de physis, tel qu'il ressort du fr. 16, il s'explicite par l'affirmation que la co-pénétration des « membres» (ta melè) entre eux, qui forment des « parties » constitutives de l'homme susceptibles d'une flexibilité constitutive d'une unité, qui est la physis se référant au même. Autrement dit, grâce au même le multiple qui est impliqué dans ce qui réfléchit (hoper phroneei) en chacun de nous est élevé au rang d'une unité, qui trouve son fondement dans l'activité de penser, qui apparaît ici sur le mode d'une unité qui est due à la plénitude propre à la physis. $\mathrm{S}$ »y ajoute la question concernant la faculté de penser (noos) et la plénitude accomplie de la pensée (noèma). Parménide dit en effet que « le plein, c'est la plénitude accomplie de la pensée » ou, si l'on préfère, « le plein est pensée accomplie » $(16,4)$. Or, c'est la plénitude réalisée par le mélange qui, par l'unité qui est sienne en tant que physis, dispose et manifeste la faculté de penser et constitue l'accomplissement de la pensée, et donc sa plénitude. Par suite, s'accordant à la plénitude de "Ce qui est le présent » d'une façon absolue et permanente $(8,24)$, parce qu'elle est régie par le même, la pensée surgit en l'occurrence comme une manifestation de la physis propre à l'homme et se donne les conditions pour accomplir pleinement sa tâche. La physis produit une plénitude, par l'unité qui est sienne.

Le caractère nouveau attribué à la notion de physis comme mélange ou unité du mélange au détriment du devenir, ébranle la physis au sens d'épanouissement, en renforçant la physis au sens de « constitution » à partir d'un multiple. Cela nous conduit à Empédocle et à Anaxagore, qui prolongent Parménide

Dans le fr. 8, Empédocle affirme: " Je te dirai autre chose encore: "d'aucune des choses mortelles, il y a épanouissement (physis), ni quelque fin dans la mort funeste, mais il y a seulement mélange (mixis) et séparation (diallaxis) de ce qui a été mélangé. L'épanouissement (physis) est la façon dont les hommes les ont nommées" ». 
Empédocle disqualifie ainsi radicalement la notion de physis, qui ne serait qu'une façon erronée utilisée par les hommes pour nommer les choses en devenir, ignorant qu'elles sont soumises à un processus de mélange et, une fois constituées comme choses mélangées, sont objet de dissociation. Pourtant cette exclusion n'est pas permanente, car au fr. 110, où il fait état d'apprentissage, il dit que les choses augmentent en nous, en disposant notre caractère (èthos), qui est la physis de chacun. Le terme physis ici ne signifie pas obligatoirement " épanouissement ", il peut signifier " constitution » issue d'un mélange, unité des constituants, comme chez Parménide. Cela veut dire qu'Empédocle est plus nuancé: il refuse l'usage de physis au sens d'épanouissement, mais consent, une fois admis le mélange et la séparation, de l'utiliser dans un sens transfiguré22. Cet infléchissement fait voir que c'est le lien entre le devenir linéaire (par succession) et l'épanouissement qui est mis en question à cette époque. Un fragment d'Anaxagore confirme cela, bien qu'il passe la notion de physis sous silence, tout en surdéterminant le terme « devenir » (ginesthai).

Comme le rapporte Simplicius ${ }^{23}$, selon Anaxagore, dont l'ouvrage s'intitulerait pourtant, comme chez la plupart des penseurs de l'époque, Peri Physeôs, le devenir et le périr sont s'assembler (composer) et se séparer (décomposer) (to ginesthai kai apollysthai sygkrinesthai kai diakrinesthai). Il aurait écrit ceci: " le devenir et le périr, les Grecs ne le conçoivent pas correctement, car aucune chose (chrèma) ne devient ni ne périt, mais c'est à partir des choses qui sont dans le présent (apo eontôn chrèmatôn) que les choses se mélangent ensemble (symmisgetai) et se séparent (se décomposent), et c'est pourquoi il faudrait qualifier plus correctement le devenir se mélanger ensemble (symmisgesthai) et le périr se séparer/se décomposer (diakrinesthai)».

Comme on le constate, les termes utilisés pour le mélange sont différents, à savoir " assembler » et " mélanger ensemble ", ce qui suscite aussitôt un difficulté quant au mélange dont il est question dans une physique du mélange et de la séparation. Déjà Parménide, on l'a vu, utilisait mixis et krasis, annonçant les grands débats du stoïcisme. Mais cela est un autre problème, qui déborde mon propos. Je dirais seulement que la troisième transfiguration de physis à laquelle adhère manifestement Anaxagore, même s'il n'utilise pas

22 Cela est confirmé par le fr. 63, où il différencie, pour le mâle et la femelle la nature (physis) de leurs membres, qui semble signifier que les dispositions du mélange forment des unités différentes, les spécifiant.

23 Simplicius, Comm. Sur la Physique d'Aristote, 163, 18. 
le terme, conçoit la notion de physis comme un mode de constitution à la suite d'un processus de mélange (au sens large du terme).

Le traité hippocratique $D u$ régime a le mérite de reprendre ce débat et de le prolonger. Tandis qu'Empédocle attribue le terme physis aux mortels, en niant le devenir et le périr, et Anaxagore parle des Grecs, Hippocrate dit que s'il emploie « devenir » et " périr », c'est à l'égard de la multitude (tôn pollôn). «Pour ma part, dit-il, j'affirme que ces processus sont en réalité « se mélanger » et « se séparer » (taûta de symmisgesthai kai diakrinesthai dèlô) $(I, 4,2)$. Cette thèse d'origine anaxagorienne insiste aussi sur le fait que rien ne devient et rien ne périt qui n'existait pas déjà auparavant, mais aussi que tout changement implique que les choses sont mélangées ou séparées (I, 4, 1). Et ajoute aussitôt un trait parménidien, en précisant que « l'homme et tous les autres animaux sont constitués de deux entités, différents par leur potentialité (dynamin), mais concordants/complémentaires dans leur activité (symphoroin de tèn chrèsin), à savoir le feu et l'eau (...) ». Le feu a le pouvoir de mouvoir et l'eau peut nourrir, et chacun domine et est dominé à son tour; ensemble ils se suffisent à eux-mêmes et à tout le reste, mais non séparés (I, 3, 1). Toutefois, Hippocrate ne se limite pas à cet infléchissement des fondements de la physique parménidienne quant aux deux principes, qu'il maintient en remplaçant la terre par l'eau, mais s'applique à accorder davantage ces processus avec la physis. C'est l'analyse du régime des hommes qui l'autorise à cette promotion de la physis, avec un sens ambivalent qui rejoint l'usage originaire d'épanouissement et de constitution, mais en fonction d'une physique du mélange et de la séparation.

Il dit que « celui qui souhaite traiter correctement du régime de l'homme doit d'abord connaître et discerner la physis de tout homme: d'une part, connaître de quels constituants il a été constitué dès l'origine et, d'autre part, discerner quelles parties prédominent ; car s'il ne connaît pas la constitution (systasin) dès l'origine, il sera incapable de connaître les effets (ta hyp'ekeinôn ginomena), et s'il ne discerne pas ce qui prédomine dans le corps, il ne sera pas capable de fournir au patient ce qui lui est utile (c'est-à-dire ce qui contribue à sa santé) ». Bien plus, il convient de connaître les aliments et boissons pour le régime, qu'ils soient conformes à la nature (physin) ou imposés par la technique humaine. Enfin, il faut connaître le moment propice d'agir (kairos) et, j'ajouterai, l'endroit du corps sur lequel il faut agir (I, 2, 1-2).

Le texte est remarquable, car il nous permet de voir, par les différentes explicitations, que l'expression pantos physin anthrôpou qu'on traduirait normalement « la nature de tout homme » ou, comme le traduit R. Joly (dans la collection Budé que je suis ici), « la nature de l'homme en général », dit 
plus que ce type de traduction. Nous y découvrons en effet la co-présence d'un processus d'épanouissement à partir de l'origine et la constitution grâce aux divers constituants et les parties impliquées. Comme nous venons d'établir que tout cela doit être interprété à partir d'une physique du mélange et de la séparation, nous pouvons conclure que la notion de physis exprime ici l'unité (comme chez Parménide) des choses qui se mélangent, mais en même temps l'épanouissement à partir du début selon un processus de constitution. Bref, nous assistons à une complexification de la notion de physis telle qu'elle avait été assumée par les penseurs d'Ionie, via Parménide et Anaxagore, qui m'autorise à la traduire en ces termes: constitution par un épanouissement qui forme progressivement l'unité de la chose. Nous verrons que lorsque Aristote, introduira la notion de forme (morphè) comme résultat (fin) d'une processus de formation, il associera l'épanouissement et les constituants du substrat qu'ils qualifie de « matière ».

\section{Les chemins vers l'institution d'une science physique}

Avant d'étudier Aristote, il convient d'aborder Platon, qui établit une quatrième transfiguration de la notion de physis, laquelle lance un véritable défi à la pensée grecque en s'attaquant au cœur même du statut matérialiste qui la régit. Cette attaque qui, nous le verrons, dénonce l'usage de la notion de physis par les écoles matérialistes, lui permet de l'utiliser abondamment dans le sens de nature d'une chose, en renforçant le sens d' " essence » qui prolonge celui de « constitution ». Les passages qui illustrent cette perspective sont très nombreux, et l'on peut trouver un inventaire empirique dans le lexique établi par Edouard des Places ${ }^{24}$. Platon considère que l'usage matérialiste de physis constitue une véritable usurpation. C'est pourquoi, je limiterai mon exposé au livre $\mathrm{X}$ des Lois, où il affronte les matérialistes et renverse leur position en situant l'Ame comme antérieure à la matière.

L'Athénien y interpelle Clinias et dit que le matérialiste envisage « le feu, l'eau, la terre et l'air comme les premiers (principes) de toutes choses, et les nomme nature ${ }^{25}$ (tèn physin onomazein), en considérant que l'âme leur est postérieure. Cette formulation nous situe, une fois encore, dans l'histoire d'une tromperie : la physis apparaît comme un nom créé par les hommes,

24 Paru dans la Collection Budé, aux Editions Les Belles Lettres, 1964, p. 559.

25 II y a ici simplification, comme si ce sont les éléments comme tels qui sont « nature », alors que « nature » signifierait en fait à la fois épanouissement à partir d'un fondement, et, à cause de la pluralité des éléments, unité de leur mélange. 
mais non plus pour remplacer le devenir par le mélange qui s'approprie le terme, mais pour situer la physis en un lieu supérieur, plus adapté à son statut propre.

Du reste Platon indique qu'il ne s'agit pas là d'un hasard, car la doctrine matérialiste ne prend pas de risque, mais soutient intentionnellement ce sens de physis. L'Athénien s'interroge: «n'avons-nous pas là, par Zeus, découvert quelque chose comme une source d'une opinion insensée où burent ceux qui ont touché aux questions concernant la physis ? » (X, 891c). Il s'agit de montrer que les matérialistes qui utilisent le nom de physis pour les éléments et les corps, non seulement se sont trompés, mais, plus grave, leur doctrine conduit à l'athéisme. Après ce que je viens de dire de l'attribution du mot physis à l'essence, il est évident que Platon ne pourrait admettre pareille dégradation. Sans le dire clairement, il insinue que le terme physis serait plus apte à exprimer l'âme plutôt que le corps. Par un retournement historique impressionnant, opposé à la réalité même, telle que je viens de l'établir depuis Homère, il considère les physiciens comme des plagiaires, alors que c'est lui le véritable plagiaire. Il est donc intéressant de voir comment il renverse le matérialisme au profit de la primauté de l'âme. De la longue argumentation qui couvre le livre X, et qui est résumée au livre XII des Lois, je pointerai quelques éléments significatifs pour voir si Platon situe la physis également dans l'ordre de l'âme, ou s'il se contente de l'utiliser pour les essences et pour ce qui est essentiel (qualifié de naturel).

Platon reproche aux matérialistes d'avoir situé l'âme et ses activités (opinion, souci, intellect, technè et loi) en dernier de la génération, alors qu'elles seraient antérieures. Ce que les matérialistes, dit-il, « veulent dire par physin, c'est la naissance (genesis) concernant les premières entités; si dès lors nous pouvons montrer que l'âme est née parmi les premières choses, plutôt que le feu et l'air, nous pourrions dire presque correctement qu'elle se distingue de tout le reste par nature (physei: entendez par "essence"). Ces considérations seront justifiées si l'on peut montrer que l'âme est plus ancienne que le corps, sans quoi elle n'existerait pas » (891e-892a). Le jeu de mots autour de physis montre un glissement entre le sens d'épanouissement et celui d'essence. Il n'empêche que Platon n'identifie pas âme et physis, mais dit seulement que l'âme diffère naturellement/essentiellement des corps. En revanche, il associe l'âme au mouvement, et développe une longue analyse des mouvements, pour aboutir à sa thèse centrale, qui prolonge le Phèdre (245c), que l'âme est automotrice.

Il pose ainsi la question: « De ce qui a pour nom "âme", quelle est la définition ? En avons-nous une autre à fournir que celle qui a été déjà 
introduite: " le mouvement capable de se mouvoir lui-même ?" ». Cela situe l'âme comme ce qui est le plus ancien de toutes choses, principe de mouvement, qui commande, tandis que le corps lui obéit conformément à la nature (kata physin) ; mieux, elle commande le corps et toutes les choses corporelles, y compris le Ciel (895e-896e). Une fois encore la notion de nature parle davantage le langage des essences que celui de l'épanouissement. C'est pourquoi, en ajoutant à l'âme ses activités, Platon vise plus haut, et la rapprocher à l'Intellect divin, ouvrant la voie non seulement vers l'irruption de la Providence divine, donc aussi de la Technè divine, déjà introduite dans le Sophiste. Cela confirme la thèse qu'il défend au livre IV des Lois, d'après laquelle « Dieu serait la mesure de toutes les choses » (716c). Or, en parlant d'Intellect divin, Platon se demande « quelle est la nature (= essence) du mouvement de l'Intellect». Il lui associe le mouvement parfait, " le mouvement circulaire uniforme et régulier autour d'un centre immuable, à des distances immuables, dans un sens et un ordre uniques » (XII, 897c-898b). Toute cette problématique nous mène loin de la notion de physis originaire, et nous rapproche davantage du sens de l' « essence ». Or, les essences, mises en rapport avec l'intellect forment les intelligibles, et dessinent les contours d'une science, celle des intelligibles. La physis au sens d'un épanouissement à partir d'un fondement un est ainsi définitivement écartée. Mais pas définitivement, car Aristote fera un saut périlleux, en changeant de cap, en revenant en partie aux origines.

Dans sa Physique, la notion de physis est étudiée au livre II, et cette étude est précédée d'une analyse générale du devenir. En cela Aristote reste fidèle à la démarche habituelle de la pensée grecque, jusqu'à Parménide ${ }^{26}$. Par cette analyse, il parvient à établir, au livre I, les principes du devenir (matière, privation et spécificité/hylè, sterèsis, eidos), avant d'accomplir un division entre différents types de devenir, distinguant surtout physis et poièsis/technè, au livre II. Comme Aristote joue sur les différents sens du terme, il est difficile de proposer une traduction précise. Pour des raisons de facilité, j'adopte la traduction habituelle « nature », que je préciserai au fil de l'exposé.

Grâce à la distinction de différents types de devenir, Aristote peut dire que " les étant par nature (physei) paraissent posséder en eux-mêmes un principe de mouvement et de stabilité, les uns selon le lieu, les autres selon la croissance et la décroissance, les autres encore selon l'altération », tandis

26 Voir mon étude «De la généalogie à la genèséologie ", dans La naissance de la raison en Grèce, éd. J.-F. Mattéi, PUF, Paris, 2006, pp. 83-96 et« Transfigurations du paradigme de la parenté ", dans Le paradigme de la parenté, éd. J. Gayon et J.-J. Wunenburger, L'Harmattan, Paris, 1995, pp. 169-186. 
que ceux qui ne sont pas constitués par nature (ta mè physei synestôta) et proviennent de la technè, reçoivent leur mouvement de l'extérieur, la seule nature qu'ils possèdent étant les matériaux issus des étants naturels, comme le bois pour fabriquer un lit $(1,192 \mathrm{~b} 8 \mathrm{ss}$.). Cette dernière précision marque une différence essentielle entre technè ancienne et technique moderne, dans la mesure où la première est proche de la nature, qu'elle imite et prolonge, tandis que la seconde met en jeu des processus multiples de provenance, éloignant l'objet technique de la nature. A telle enseigne que Heidegger ait pu déduire que par la technique moderne il y a occultation de l'entrée dans la présence (de la physis $)^{27}$. Cette orientation de la lecture du texte, aussi intéressante qu'elle soit, occulte l'analyse principielle visée par Aristote en vue de fonder une science physique, plutôt qu'un discours sur la nature.

Cela ressort déjà par les trois mouvements qu'Aristote retient, et dont la physis en est la condition. Ils concernent des catégories secondaires (respectivement le lieu, la quantité et la qualité), mettant provisoirement entre parenthèses le devenir selon l'étance (ousia), qui se limite à la naissance/ génération (genesis) et au périssement (phtora). Or, lorsqu'on tient compte ainsi de la génération, celle-ci devient un obstacle pour traduire physis par " épanouissement », qui ne concerne que le devenir conforme à l'étance (ousia). Le terme physis mis en rapport avec les mouvements qu'elles met en œuvre semble plus proche d'une constitution dynamique. Pourtant, la physique ne se constitue comme science qu'en tant qu'elle étudie d'abord les étants naturels distincts, c'est-à-dire les étances (ousiai), et qui ne se limitent pas à la spécificité qu'on rencontre différemment dans chacun des trois mouvements, mais, au contraire, déploient leur spécificité propre selon un processus de formation qui fait émerger la forme (morphè) en tant que manifestation spatiotemporelle de la spécificité. La forme, une fois constituée, exprime la fin et la finalité de l'étant, et, de ce fait, devient en même temps origine de toutes les activités concernant l'étance en question.

Toutefois, au départ de son analyse, Aristote dit, d'une façon elliptique, ceci: " nous avons dit ce qu'est la nature [comme origine]; et possède une nature [comme constitution] (physin) tout ce qui possède un tel principe. Et toutes ces choses sont étance, car toujours la nature (hè physis) est un certain sujet et réside dans un sujet (hypokeimenon gar ti, kai en hypokeimenôi estin)»

27 Sur cette question voir mon étude «Technè ancienne et technique moderne selon Heidegger », Revue de philosophie ancienne, 4, 1986, pp. 253-297. La thèse heideggerienne peut être prolongée et corrigée par d'autres éléments, comme celui de la technico-économie et le problème du risque : voir mon livre $L a$ proximité et la question de la souffrance humaine, Ousia, Bruxelles, 2005, pp. 667 ss. 
(192b32-34). Cette dernière assertion se comprend seulement si l'on se souvient, d'une part, que l'étance est sujet ultime de toute prédication et, comme telle, une entité pour ainsi dire absolue se constituant par une génération et, d'autre part, que la physis, étant une origine de mouvements selon des catégories, lui est intrinsèque, c'est-à-dire elle est dans un sujet. Nous découvrons ici le lien de la physis avec la matière, dans la mesure où le sujet, une fois qu'il a été analysé au livre I relativement au devenant (to gignomenon), prend le nom de « matière » (hylè). Mais, en même temps, par l'expression «dans un sujet», on découvre que quelque chose d'autre, différent du sujet, donc de la matière, l'excède. En l'occurrence, c'est la physis qui prétend à ce plus. Du reste, déjà, au livre I, Aristote nous apprend qu'à l'inverse de la pensée ionienne, ce n'est pas l'ousia matérielle comme telle qui s'épanouit en tant qu'elle est physis, faisant des activités (comme la technè) et configurations des affections et des épiphénomènes, mais qu'à côté de ce fonds matériel, il y a d'autres principes équivalents ou plus importants, comme la privation et la spécificité. En distinguant, au livre II, physis et technè, il fait voir que la technè est bien différente de la matière, ce qui permet de voir maintenant, plus clairement que de la même façon la physis qui ne concerne que l'étance naturelle est certes immanente, mais sauvegarde sa différence avec le sujet/matière. On peut supposer que c'est la spécificité (eidos) apportée par l'un et l'autre de ces processus, qui marque la différence avec la matiuère, et, de ce fait, constitue ce qui subvertit le point de vue des Ioniens. Du même coup on comprend aussi que c'est la physis au sens d' « épanouissement à partir d'un fondement un » qui est ébranlée, en laissant ouverte une transfiguration qui l'investit par l'idée d'une « constitution », éclairée par les notions de spécificité (eidos) et de forme (morphè). Mais, en réalité, les choses sont encore plus complexes, car, qu'il s'agisse de technè ou de physis, la spécificité intègre la matière par l'action de la cause efficiente en tant qu'elle meut (to kinoun) et, transmettant la spécificité, celle-ci transforme la matière pour produire soit une œuvre (ergon), soit réaliser une forme (morphè).

Cette complexification me permet de dire qu'en dépit de sa transfiguration, la physis aristotélicienne conserve la dualité originaire (épanouissement et constitution) mais à travers deux variables nouvelles: la spécificité et le mouvement, d'origine platonicienne - mais dont Platon avait dissocié de la physis, soit parce qu'il la dévaluait aussi longtemps qu'elle était l'apanage des penseurs matérialistes, soit, au contraire, parce qu'il lui conférait une promotion inattendue en la situant sur le plan des essences transcendantes.

En partant établissant la définition de la physis, Aristote s'est permis de faire un pas de plus et d'élucider la sémantique du terme. Il précise ainsi que 
sont qualifiées de « conforme à la nature (kata physin) » toutes ces choses, mais aussi leurs attributs (propriétés) essentiels, par exemple le fait que le feu est transporté vers le haut, « car cela n'est pas nature ni ne possède de nature, mais est « par nature » et conformément à la nature » (192b35-193a1). Aristote éclaire ici un usage fréquent chez les penseurs grecs, et surtout chez Platon, où règne pourtant une forme l'opacité à travers le thème de l' " essence ». Il apparaît, d'abord, que ce qui n'est pas de l'ordre de la production, de l'action, du hasard, etc. mais concerne des étants naturels, peut être qualifié par nature ou conformément à la nature. Mais il apparaît, ensuite, que les propriétés de ces étants (qualifiés d'attributs ou accidents essentiels, ou propres) peuvent bénéficier du même usage, perturbant l'usage univoque de ces expressions. Or, ces deux perspectives appartiennent aux données épistémologiques pour former une science, ce qui montre que, bien avant qu'Aristote établisse les causes, il a déjà fait un pas décisif vers l'institution d'une science de la nature, et non plus seulement du devenir grâce au trois principes. Le passage d'une problématique générale du devenir vers une problématique particulière de la nature met en œuvre des éléments supplémentaires qui cernent de plus près le devenir des étants naturels. Il convient de noter ici le parallélisme avec ce que j'ai établi pour Homère, où le contexte généalogique précède l'utilisation de la nature, limitée aux plantes (arbres), rendant une plus grande consistance à la sémantique du phyô. Avec Aristote le rapport devient plus étroit entre le devenir et le devenir naturel (physis), moyennant les différentes médiation amorcée par les Ioniens jusqu'aux manipulations platoniciennes.

Face à ces jeux subtils, il reste à voir comment Aristote confère à la notion de morphè un lien plus grand avec la physis. Pour ce faire, il fait un pas supplémentaire en distinguant une nature comme la matière prochaine, sujet/substrat des choses qui possèdent en elles un principe de mouvement et de changement et une nature comme forme, c'est-à-dire comme spécificité conforme à la raison d'être de la chose (tèn morphèn kai to eidos to kata ton logon) (193a28-31). L'irruption de la forme situe l'épanouissement de l'étant en rapport avec l'ordre de la finalité. C'est que l'épanouissement est compris, par Aristote, comme une naissance qui se déploie comme " un chemin vers une nature », de sorte que « ce qui s'épanouit atteint quelque chose en tant qu'il s'épanouit » (193b11-18). C'est dire qu'au fil de son analyse le sens d'épanouissement retrouve sa puissance ancienne, mais avec un plus: d'abord, la mise en œuvre d'une finalité exprimée à travers la physis comme forme (hè morphè physis), qui révèle un étant constitué avec ses constituants (193b1112); ensuite, la physis comme une fin (ce qui est ultime) et ce en vue de quoi (hè physis telos jai ou heneka), qui intègre le point de vue de la «constitution». 
L'ensemble de ces perspectives réussissent à délimiter trois des quatre causes: la cause matérielle (hylè), la cause dite formelle (eidos) et la cause finale (morphè comme ou hou heneka). La cause efficiente, to kinoun est introduite surtout au livre III, consacré au mouvement.

En effet, au début du livre III, Aristote dit que si la physis est un principe de mouvement et de changement, et comme, d'autre part, la recherche concerne la physis, il ne faut pas perdre de vue ce qu'est le mouvement, car si on l'ignore, on ignore du même coup la physis. L'analyse qu'il propose étudie le mouvement en général, avant même qu'il soit question du mouvement propre aux étants de la nature. Cette étude qui ressemble à celle du devenir au livre I, qui précède celle de la nature au livre II, cherche, en amont, à rendre le mouvement indépendant de la physis, dans la mesure où il peut appartenir à des réalités qui ne sont plus de l'ordre de la nature, comme les sphères du monde supralunaire, où domine le premier mouvement, circulaire et parfait qui se meut par lui-même, — rencontrant ainsi les prérogatives que Platon avait attribuées à l'âme. En revanche, la physis, suppose toujours une cause efficiente interprétée selon le mouvement (le mouvant, ce qui meut: to kinoun) qui transmet la spécificité. Une fois cette spécificité transmise à travers le mobile (sujet, matière étant en puissance) qui est aussitôt mû, l'étant naturel acquiert son autonomie, dans la mesure où la physis est un principe de mouvement, de changement et de stabilité. Cependant, ces données ne constituent pas le dernier mot d'Aristote, car il introduit, en aval, la génération spontanée (automaton genesis), qui reçoit les conditions de son déploiement des facteurs intrinsèques de la matière (la putréfaction) et des facteurs extérieurs (la chaleur du soleil et les conditions de l'environnement). Ce processus subvertit à la fois la physis du monde sublunaire et le mouvement céleste du monde supralunaire. De sorte que tout se passe comme si la physis aristotélicienne, bien qu'elle associe épanouissement, engendrement, constitution et mouvement, se laisse déborder par deux processus concurrents: le devenir spontané et le mouvement circulaire céleste. C'est cela qui permet à Aristote de dire, dans sa Métaphysique, qu'il existe quelqu'un de supérieur au physicien, car la nature (physis) est un certain genre de l'étant, et non le tout ${ }^{28}$.

Cette analyse de la notion, de physis pourrait être prolongée dans différentes directions dans l'œuvre d'Aristote. Une première direction est celle des différents sens du terme physis dans le livre Delta, 4. On y trouve une grande partie de ce que j'ai retenu ci-dessus à partir de la Physique, mais 
aussi d'autres précisions ${ }^{29}$. La donnée la plus intéressante se trouve dans le dernier sens retenu, où Aristote porte l'accent sur le lien entre physis et ousia, et qui entraîne, dans son sillage, d'autres sens seconds, comme la matière et l'épanouissement: « d'après ce qui vient d'être dit, la physis première, qui est aussi qualifiée de principale, c'est l'étance (ousia) des choses qui possèdent un principe (archèn) de mouvement en elles et en tant que tellee; car la matière qui est réceptrice de ce principe est qualifiée [aussi] physis, et les devenirs et le fait de s'épanouir (phyesthai) qui provient de ce principe sont des mouvements. Et le principe du mouvement des étants naturels est cette physis, qui leur est d'une certaine façon immanente soit en puissance, soit en entéléchie » (1015a13-19). Autrement dit, peuvent être qualifiés de physis l'étance naturelle (sens premier et principal), le principe des mouvements en elle et la matière. Cela fait voir que le sens premier n'est pas le principe immanent du mouvement d'une étance naturelle, mais l'étance naturelle ellemême. Et, en plus, une fois encore la notion de mouvement est envisagé comme différente de la nation de nature, ce qui montre qu'aussi bien la cause efficiente (le mouvant, to kinoun) que les mouvement céleste ne sont pas de l'ordre de la physis.

Une deuxième direction à analyser est celle des ouvrages dits de biologie, où les quatre causes sont excédées par des processus successifs et variés dans le déploiement des animaux (Aristote parle de génération, ce qui oriente clairement le débat vers un aspect de la physis). La multiplicité des processus, nous rapproche davantage de la problématique de la provenance complexe, que la théorie des quatre cause cherche à simplifier pour parvenir à établir une science qui soit intelligible.

Une troisième direction concerne la différence entre physique et mathématiques comme sciences (Phys. II). Elle révèle que, non seulement l'intelligibilité mathématique est de l'ordre de l'abstraction et concerne une catégorie seconde (quantité), mais que la physique, pour étudier ce qui est le propre d'un étant naturel, doit éviter toute forme de constructions intermédiaires (mathématiques, paradigmes, métaphores, mythes) qui serait le défaut du Timée, et qui ne parviennent jamais à approcher le réel comme tel.

Une quatrième direction est celle qui situe la question de l'âme par rapport à la physis, et qui, par la problématique de l'intellect, nous situe déjà au delà de la physique proprement dite, en nous introduisant dans le domaine de la métaphysique, et notamment dans l'ordre agathologique, où l'intellect et dieu 
sont considérés comme des modes de l'étance relativement au Bien (Ethique à Nicomaque, livre I).

Enfin, une quatrième direction concerne le rapport entre spécificité et genre (genos), qui nous situe d'emblée dans la sémantique de la parenté, survivance de la pratique généalogique du mythe, encore présente dans la façon dont Platon déploie sa méthode de division dans le Sophiste, le Politique et le Philèbe $e^{30}$.

En guise de conclusion de cette mini-odyssée à travers un des itinéraires sinueux parcouru par la notion de physis dans la pensée anciennejusqu'à Aristote, je me permets de résumer le chemin suivi. Pour établir les transfigurations de la physis au cours de cette histoire, j'ai commencé par circonscrire l'émergence de la notion de physis dans le monde archaïque, chez Homère, où le sens d'épanouir semble dominer. Puis, j'ai tenté de repérer comment s'est accompli le passage à la pensée ionienne (représentée par Anaximandre et Héraclite), où la physis comme épanouissement avait acquis un sens plus étendu, en investissant le fonds propre des choses, dans le sens d'un épanouissement à partir d'un fondement un. La critique des Ioniens, par Parménide, a permis d'irruption d'un sens nouveau lié au mélange, avec le renforcement du sens de « constitution ». Cette deuxième transfiguration de la notion de physis, a abouti à sa disqualification par Empédocle et Anaxagore, corrigée en partie par Hippocrate dans le Régime, qui à réuni les sens d'épanouissement et de constitution. A cette troisième transfiguration a succédé une quatrième, grâce à Platon, qui, en disqualifiant la sens matérialiste des origines et en conférant le mouvement à l'Ame, il situe la physis surtout dans le domaine des essences, sous-déterminant son caractère d'épanouissement au profit de celui de stabilité. Par là, il déplace le mouvement créatif du côté de l'Ame automotrice qui absorbe la physis au profit de l'Art divin. Enfin, le retour aux penseurs ioniens s'accomplit par Aristote. Il infléchit le sens originel en l'investissant de données platoniciennes, ce qui l'autorise à réaliser une nouvelle conception de la physis, dominée par la différence entre matière et spécificité (eidos) en vue de réaliser la forme (morphè) comme expression de la finalité. Toutefois, pour préserver l'autonomie du mouvement, Aristote distingue physis et mouvement (kinèsis) qu'il situe tant du côté de la cause efficiente (du mouvant, to kinoun) que du côté du premier mouvement appartenant au monde supralunaire. En même temps, il introduit, dans le monde sublunaire où la physis domine, la possibilité d'une génération spontanée, ce qui confirme que la notion de 
devenir déborde à son tour celle de physis, ne serait-ce que parce que les devenirs sont multiples, et la physis n'exprime qu'un devenir parmi d'autres, comme la production, le hasard, la génération spontanée et jusqu'à un certain point l'action. Pour terminer, je peux ajouter que c'est cette cinquième transfiguration qui rend possible l'avènement d'une science du sensible et du devenir, et qui consacre définitivement le terme " physique », compris dans le sens d'une " science physique », quel que soit le type de science de la nature que cette expression recouvre aujourd'hui. 